\title{
Interaction morphology and bond strength of nanofilled simplified-step adhesives to acid etched dentin
}

Vinicius Di Hipólito ${ }^{1}$

André Figueiredo Reis ${ }^{2}$

Sumita B. Mitra ${ }^{3}$

Mario Fernando de Goes ${ }^{4}$

\section{ABSTRACT}

Objective: To evaluate the effect of nanofillers incorporated into adhesives on the microtensile bond strength ( $\mu$-TBS) and interfacial micromorphology to dentin.

Methods: The occlusal enamel of 5 human molars was removed and each tooth sectioned into four quarters. The exposed dentin was treated with one of the following adhesives: Adper Single Bond (SB-unfilled), OptiBond Solo Plus (OS-barium aluminoborosilicate, 400nm $\emptyset$ ), Prime \& Bond NT (NT-colloidal silica, 7-40 nm Ø) and Adper Single Bond 2 (SB2-colloidal silica, 5nm Ø). Cylinders of resin-based composite were constructed on the adhesive layers. After 24-hour storage, the restored tooth-quadrants were sectioned to obtain stick-shaped specimens $10.8 \mathrm{~mm} 2$, cross-sectional areal and submitted to $\mu$-TBS at a cross-speed of $0.5 \mathrm{~mm} / \mathrm{min}$. Data were analyzed using one-way ANOVA and Tukey's test (alpha $=.05$ ). Twenty-eight additional teeth were used for interfacial micromorphologic analysis by SEM (16-teeth) and TEM (12-teeth). The dentin surfaces of 32 discs were treated with the adhesives ( 8 discs for adhesive) and laminated to form disc-pairs using a flowable resin composite for SEM/EDS analysis. For TEM, $90 \mathrm{~nm}$-thick nondemineralized unstained sections were processed.

Results: SB2 showed significant higher bond strength than SB, OS and NT. The SEM/EDS and TEM analysis revealed nanofillers infiltrated within the interfibrillar spaces of the SB2-hybrid layer. Fillers were concentrated around patent tubular orifices and in the adhesive layer for OS and NT.

Conclusion: The presence of nanofillers within the interfibrillar spaces of the SB2-hybrid layer suggests its importance in the improvement of the $\mu$-TBS. (Eur J Dent 2012;6:349-360)

Key words: Simplified-step adhesives; bond strength; nanotechnology; SEM; TEM.

1 Biomaterials Research Group, School of Dentistry, Anhanguera-Bandeirante University of São Paulo UNIBAN, São Paulo, SP, BRAZIL

2 Dental Research and Graduate Studies Division, Department of Restorative Dentistry, Guarulhos University, SP, BRAZIL

3 Mitra Chemical Consulting LLC, 1952 Nature View Lane, W.St. Paul, MN 55118, USA

(Former address: Corporate Scientist, 3M ESPE Dental Products Division - 3M Company St. Paul, MN, 55144, USA)
4 Department of Restorative Dentistry, Piracicaba School of Dentistry, University of Campinas, Piracicaba, SP, BRAZIL

- Corresponding author: Dr. Vinicius Di Hipólito Rua Maria Cândida, 1.813, Vila Guilherme, São Paulo, SP. CEP: 02071-013 BRAZIL

Tel: +55 1993307868 Fax: +55 1129679077 Email: vdhipolitodyahoo.com.br 


\section{INTRODUCTION}

The adhesion of the contemporary dentin bonding agents is essentially micromechanical in nature. It is the result of the resin monomer infiltration into the demineralized collagen network and their polymerization in situ. This interaction forms a new micromorphologic structure referred to as a hybrid layer or interdiffusion zone. ${ }^{1-2}$

A fundamental condition for the formation of the hybrid layer is the maintenance of the dentin organic matrix hydrated after demineralization, which supports the expansion of the collagen fibrils and preserves the integrity of the interfibrillar spaces. This disposition is compatible with the bonding process, since it allows an appropriate infiltration of the resin monomers and may be achieved through the application of the total-etch wet-bonding technique associated with resin monomers dissolved in non-aqueous organic solvents or an aqueous solution of hydrophilic primers. ${ }^{3}$ Alternatively, the self-etch bonding technique uses acidic monomers that combine tooth surface etching and priming in a single procedure, minimizing the risk of technical imperfections. ${ }^{4}$

When the dentin is demineralized by means of the wet-bonding technique, there is a substantial reduction in the mechanical properties of the remaining structure, ${ }^{5}$ particularly when the collagen network remains expanded in the aqueous medium. ${ }^{6-7}$ This condition of transitory fragility is partially reversed with the infiltration of resin monomers inside the collagen network, as originally demonstrated in an experimental macro-model of the hybrid layer ${ }^{5}$ and a hypothetical device. ${ }^{8}$ The results of these studies demonstrated a positive correlation between the ultimate tensile bond strength of the adhesive resin and that of the resin-infiltrated demineralized dentin, suggesting that stronger resins may result in hybrid layers that are more resistant to mechanical efforts.

Based on these evidences, a tendency exists to incorporate inorganic fillers in adhesive systems in the attempt to improve the bonding effectiveness. Initially, the fillers used were micrometric sized. However, they are much larger when compared with the interfibrillar spaces of the demineralized dentin, ${ }^{9}$ which have been reported to be about $12 \mathrm{~nm}$ in width. ${ }^{10}$ So, the fillers were normally distributed in the adhesive layer and around the tubular orifices at the bonding interface, but not inside the hybrid layer. ${ }^{9}$

With the progressive development of the den- tin bonding agents, an innovative simplified-step adhesive, Prime \& Bond NT (Dentsply, Caulk, Milford, OF), was introduced that incorporates the use of inorganic fillers comprised of fumed silica with primary particles of nanometric dimensions. Even with the reduced primary particle size of $7 \mathrm{~nm}$, the nanofillers did not infiltrate the collagen network of the etched dentin. ${ }^{11}$ On a nanometric scale, nanofillers have the natural tendency to aggregate/agglomerate, forming micrometric electro-dense clusters that are larger than the interfibrillar spaces. ${ }^{11}$ As a result, the strengthening of the hybrid layer claimed by the manufacturer was not observed and the resulting bond strength was described as being at the same level as other simplified-step adhesives without nanofillers. ${ }^{12-13}$

More recently, a new simplified-step adhesive, Adper Single Bond 2 (3M ESPE Dental Pruducts Division St. Paul, MN, USA) was introduced. This adhesive contains nanofillers of silica with $5 \mathrm{~nm}$-size. The nanoparticles are treated in situ with a silane stabilizing agent during manufacture. According to the manufacturer, the improvement in the technology used for surface treatment of the nanofillers provides higher stability and avoidance of aggregation/agglomeration. This way, the nanofillers could remain uniformly dispersed and would be able to infiltrate the interfibrillar spaces.

As little information is available on this topic, the aim of the present study was to investigate the interaction morphology and bond strength of four simplified-step adhesives applied to acid etched dentin: two of them with nanofillers, one with microfillers and the other one without fillers (control). The tested hypothesis was that there is no significant difference between the filled and the unfilled adhesives evaluated regarding the interaction morphology and bond strength to dentin.

\section{MATERIALS AND METHODS}

Thirty-three caries-free, human third molars were collected according to the local Institutional Review Board (\# 073/2007), with the informed consent of the donors. They were stored in a $1 \%$ chloramine $\mathrm{T}$ solution at $4^{\circ} \mathrm{C}$ and used within one month following extraction.

\section{Bonding Procedures}

The three simplified-step filled adhesives examined were OptiBond Solo Plus (OS), Prime \& 
Bond NT (NT) and Adper Single Bond 2 (SB2). In addition, the simplified-step unfilled adhesive Adper Single Bond (SB) was used as a control. The components, batch numbers and manufacturers' recommended protocols of the tested adhesives employed are summarized in Table 1. Prior to bonding procedures, the exposed middle dentin surfaces were wet-polished with 600-grit silicon carbide paper under running water to create a standard smear layer. The 35\% phosphoric acid with silica thickener (ScotchBond ${ }^{\mathrm{TM}}$ Etchant, 3M ESPE Dental Products, St. Paul, MN) was used to etch the dentin surfaces. After that, the adhesives were applied following the respective manufactures' instructions (Table 1) and light-cured using a quartz-tungsten-halogen curing unit (XL 3000 $3 M$ ESPE) with a power density of $600 \mathrm{~mW} / \mathrm{cm}^{2}$.

\section{Microtensile Bond Testing}

Five teeth were used. After removal of the occlusal enamel and the roots, each tooth received two longitudinal sections perpendicular to each other (mesiodistally and buccolingually) in order to produce four quarters using a diamond-impregnated disk (Extec, Enfield, CT, USA) under water cooling in a specific cutter machine (Isomet 1000, Buehler, Lake Bluff, IL, USA). After that, the dentin surface of each quarter from the same tooth was individually treated with one of the adhesives evaluated. Subsequently, three 2-mm-thick increments of a resin composite (Filtek ${ }^{\mathrm{TM}}$ Z250, $3 \mathrm{M}$ ESPE) were built up on each bonded surface and individually light-cured for $20 \mathrm{~s}$, constituting a crown of $6.0 \mathrm{~mm}$ in height. The restored toothquadrants were stored in distilled water at $37^{\circ} \mathrm{C}$ for $24 \mathrm{~h}$. After this period of storage, they were serially sectioned in a longitudinal direction into 0.8-mm-thick slabs, using a diamond saw. Each slab was further sectioned to produce bonded specimens or sticks of approximately $0.8 \mathrm{~mm}^{2}$ in cross-sectional area. The sticks were individually fixed to a custom-made testing jig (Geraldeli's device) ${ }^{14}$ with a cyanoacrylate glue (Model Repair II Blue, Dentsply-Sakin, Japan) and subjected to tensile load at a crosshead speed of $0.5 \mathrm{~mm} /$ min until failure (Instron 4411, Instron Corporation, Canton, MA, USA). After testing, the dentin and composite sides of the failed specimens were sputter-coated (40 mA for $120 \mathrm{~s}$ ) with gold/palladium (SCD 050; Balzers, Schaan, Leichtenstein) and examined through scanning electron microscopy (SEM) (JSM 5600LV; JEOL, Tokyo, Japan) with $85 \mathrm{x}$ magnification, operating at $15 \mathrm{kV}$, high vacuum and secondary electron mode. No pre-testing failures occurred. Failure modes were classified in one of the four following categories: $:^{15}$ type I: cohesive failure in the adhesive; type II: cohesive failure in the dentin; type III: cohesive failure in the hybrid layer; and type IV: mixed failure: cohesive in the adhesive and hybrid layer. In cases of uncertainty, examination under higher magnifications (1500-3000x) was performed in order to confirm the nature of the failure. Statistical differences between the mean bond strength of the four bonding systems tested were submitted to one-way ANOVA and Tukey's HSD post-hoc test, at a pre-set alpha

Table 1. Materials used in this study.

\begin{tabular}{|c|c|c|c|}
\hline Adhesive Systems & Components & Batch number & $\begin{array}{l}\text { Manufacturers' Recommended } \\
\text { Protocols }\end{array}$ \\
\hline $\begin{array}{l}\text { Adper Single Bond ( } 3 \mathrm{M} \\
\text { ESPE, St. Paul, MN,USA) }\end{array}$ & $\begin{array}{l}\text { Bis-GMA, HEMA, dimethacrylates, methacrylated poly- } \\
\text { alkenoic acid, copolymer, initiators, water and ethanol. }\end{array}$ & 20000630 & a (15s); b (10s); c; d; h (5s), i (10s) \\
\hline $\begin{array}{l}\text { OptiBond Solo Plus (Kerr } \\
\text { Corp. Orange, CA, USA) }\end{array}$ & $\begin{array}{l}\text { Bis-GMA, HEMA, GPDM, initiator, ethanol, fumed silica, } \\
\text { barium aluminoborosilicate and sodium hexafluorosili- } \\
\text { cate. }\end{array}$ & 14088 & $\begin{array}{c}\text { a (15s); b (10s); c; e (15s); h (3s); } \\
\text { i (20s) }\end{array}$ \\
\hline $\begin{array}{l}\text { Prime \& Bond NT (Dentsp- } \\
\text { ly Caulk, Milford, DE) }\end{array}$ & $\begin{array}{l}\text { UDMA, PENTA, R5-62-1 resin, T-resin, D-resin, butylated } \\
\text { hydroxitoluene, EDMAB, cetylamine hydrofluoride, initia- } \\
\text { tor, stabilizers, acetone and fumed silica nanofillers. }\end{array}$ & 306000197 & $\begin{array}{c}\text { a (15s); b (10s); c; f; g (20s); h (5s); } \\
\text { i (10s) }\end{array}$ \\
\hline $\begin{array}{l}\text { Adper Single Bond } 2 \text { (3M } \\
\text { ESPE, St. Paul, MN, USA) }\end{array}$ & $\begin{array}{c}\text { Bis-GMA, HEMA, dimethacrylates, methacrylated poly- } \\
\text { alkenoic acid, copolymer, initiators, water, ethanol and } \\
\text { silane-treated silica nanofillers. }\end{array}$ & 100076 & a (15s); b (10s); c; d; h (5s), i (10s) \\
\hline
\end{tabular}

Abbreviations: Bis-GMA: bisphenol-glycidyl methacrylate; HEMA: 2-hydroxyethyl methacrylate; GPDM: glycerol phosphate dimethacrylate; UDMA: Urethane dimethacrylate; PENTA: phosphonated penta-acrylate ester; EDMAB: Ethyl 4-dimethyl amino benzoate. Application techniques - a: acid etching; b: rinsing; c: excess moisture removed from the preparation using a cotton pellet; $d$ : application of two consecutive coats of adhesive; e: aplication of one coat of the adhesive; f: application of three consecutive coats of adhesive; $g$ : the surface should remain fully wet; $h$ : gently air-drying; i: light-cure.

Note: The brand name of Adper Single Bond 2 is used in Latin America and Oceania, while Adper Scotchbond 1 XT is used in Europe, Adper Single Bond Plus in the USA and Adper Single Bond $1 \mathrm{XT}$ in South Africa. 
of 0.05 . The statistical unit was beams, not teeth.

Scanning electron microscopy and energydispersive X-ray

Sixteen teeth were used, randomly divided into four groups. The roots were severed along the cement-enamel junction using a diamondimpregnated disk under water cooling, followed by the removal of the occlusal enamel through a second parallel section. Two discs, of $1 \mathrm{~mm}( \pm 0.5)$ in thickness, were prepared by means of two parallel sections of the remaining coronary portion. The same adhesive system was applied to the adjacent dentin-surfaces. The discs were laminated into disc-pairs using a thin layer of the flowable composite FilteK Flow (3M ESPE), according to the sandwich-technique first described by Inokoshi et al. $^{16}$ The dentin-resin-dentin sandwiches were light-cured for $160 \mathrm{~s}$ in four different directions and stored in distilled water at $37^{\circ} \mathrm{C}$ for $24 \mathrm{~h}$. Following that, each sandwich was mesio-distally sectioned to produce 4 resin-dentin bonding interfaces for evaluation. Common procedures were employed to specimen preparation for SEM examination of the bonding regions, including demineralization in $50 \%$ phosphoric acid for $5 \mathrm{~s}$, deproteinization by immersion in $10 \% \mathrm{NaOCl}$ for $15 \mathrm{~min}$, dehydration in ascending ethanol series $(20 \%, 30 \%, 40 \%, 50 \%$, $70 \%, 90 \%$ and $100 \%$ for at least 20 min per step) and immersion in hexamethyldisilazane (HMDS) for 10 min. ${ }^{17}$ After chemical dehydration, half of the processed bonding interfaces were sputter coated (40 mA for $120 \mathrm{~s}$ ) with gold/palladium (SCD 050; Balzers) in order to examine the resin-dentin interfaces in a SEM (JSM 5600LV; JEOL) operated in high vacuum and secondary electron mode. The other half of the processed bonding interfaces were submitted to carbon evaporation ISCD 050;
Balzers) for energy-dispersive X-ray (EDS) analysis using a SEM (JSM 5600LV; JEOL) equipped with an X-radiation detector EDS (Voyager, Noran Instruments), operated in low vacuum and backscattered electron mode. This EDS equipment contains an ultra-thin Norvar window and works with a Windows NT-based (Vantage) digital microanalysis system. A qualitative and semi-quantitative elemental analysis was performed in four different points of each specimen lpoint 1 - adhesive layer; point 2 - hybrid layer; point 3 - initial portion of the resin tag; point 4 - medium third of the resin tag). The working distance (WD) was $20 \mathrm{~mm}$ and the accelerating voltage was $15 \mathrm{kV}$ for both SEM and EDS analysis. A schematic representation of the specimens' preparations is showed in Figure 1.

\section{Transmission electron microscopy}

Twelve teeth were used. The occlusal enamel was removed to expose a flat of middle dentin. After the bonding procedures were performed, a thin layer $(0.5 \mathrm{~mm}$-thick) of flowable composite (Protect Liner F, Kuraray Medical Inc., Tokyo, Japan) was applied to the top of the adhesive layer. The resin-dentin bonding interfaces were exposed and processed according to the protocol previously described by Tay et al $^{11}$ The resulting epoxyresin embedded, $90 \mathrm{~nm}$-thick nondemineralized unstained sections, were examined by means of transmission electron microscopy (TEM) (EM10 Zeiss, Oberkochen, Germany) operating at $80 \mathrm{kV}$.

\section{RESULTS}

Bond strength results and failure analysis

The means and standard deviations (SD) of the microtensile bond strength ( $\mu$-TBS) test for the adhesives evaluated are presented in Table 2. No significant differences were found between the re-

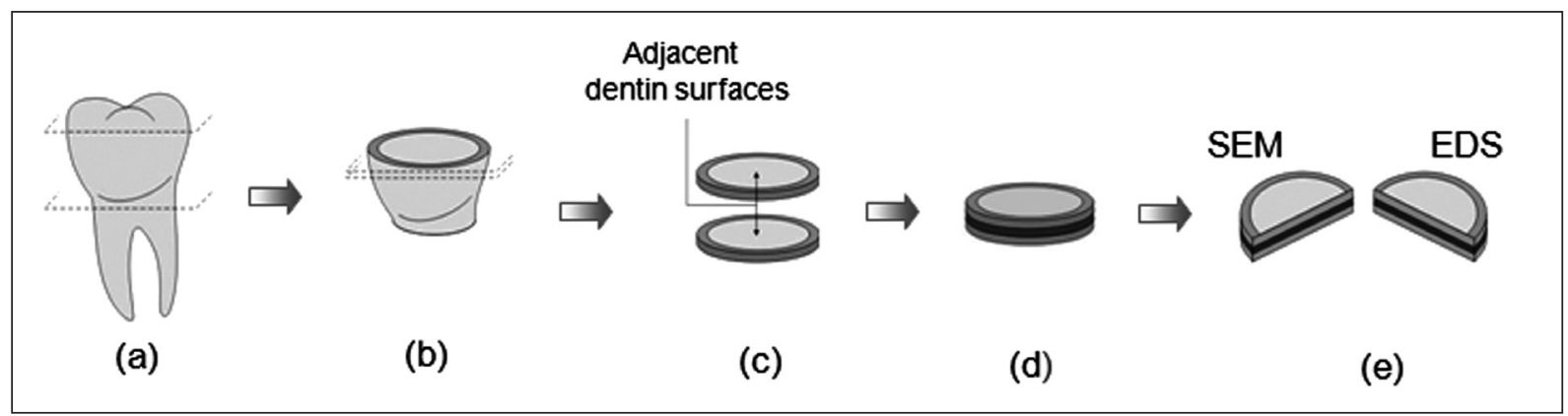

Figure 1. Schematic representation of the specimen preparation for SEM and EDS analysis. (a) The occlusal enamel of the molar crown and the root portion were sectioned. (b) Two parallel sections were made on the remaining tooth. (c) The adjacent flats of dentin of the discs were each treated with the same bonding system. (d) A "sandwich" block of dentin-resin-dentine was made, laminating the disc-pairs with a low viscosity resin. (e) The block was mesio-distally sectioned producing 2 bonding interfaces in each half-block. 
sults of SB, OS and NT (P>.05). The $\mu$-TBS mean value of SB2 was significantly higher than those obtained for the other adhesives $(P<.05)$.

The failure pattern distribution (\%) as analyzed by SEM can be observed in Figure 2. There was a predominance of the type IV failure mode for the adhesives tested. SB2 presented the smallest percentage of the type III failure mode and the largest incidence of the type I failure mode. All of the adhesives evaluated showed similar percentages of the type II failure mode.

Scanning electron microscopy and energydispersive X-ray analysis

Representative SEM micrographs of the bonding region of the adhesives studied are illustrated in Figure 3, where authentic hybrid layers about $5 \mu \mathrm{m}$-thick were observed. The bonding region of SB (Figure 3a), OS (Figure 3b) and NT (Figure 3c) showed similar micromorphological aspects, characterized by uniform hybrid layers and resin tags distributed along the interface. Figure $3 \mathrm{~d}$ illustrates the granular aspect of the hybrid layer formed by SB2. Circular formations, which prob-

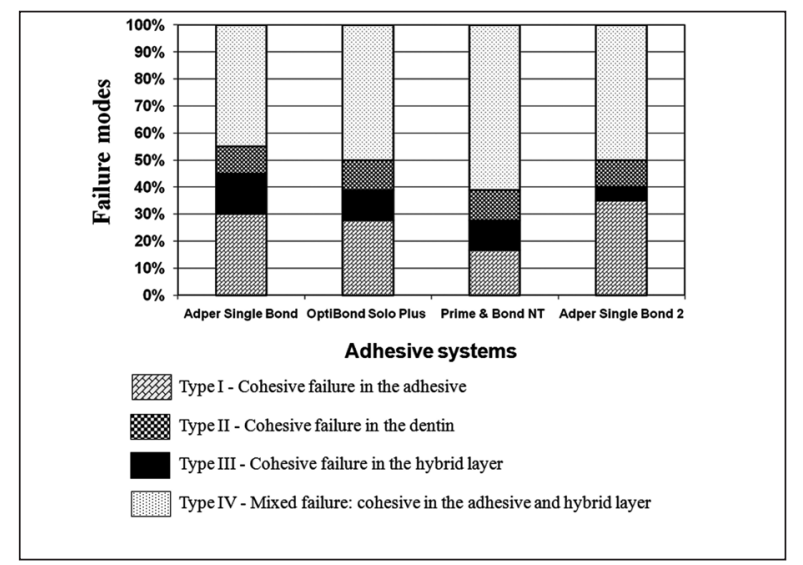

Figure 2. Graphed presentation of proportional prevalence of fracture modes for the adhesives evaluated.

ably represent a grouping of the polialkenoic acid molecules, could be identified in the adhesive layer. Another particularity of this bonding region was the presence of round grains in the medium third of some resin tags.

A qualitative and semi-quantitative elemental analysis of a point selected at the bonding region

Table 2. $\mu$ TBS means $( \pm \mathrm{SD})$ in MPa for the adhesives evaluated formed by the adhesive systems is shown in Figure 4. Carbon, evaporated to coat the specimens, was always present. Figure 4 a shows a representative elemental distribution of the selected point 2 at the bonding region formed by SB. Calcium and phosphorus were primarily detected. Chlorine and oxygen were identified in reduced amounts. As was expected, other chemical elements, which could suggest the presence of inorganic filler, were not detected in any of the other selected points.

The chemical elements calcium and phosphorus were predominant in the initial portion of the resin tags of the bonding region formed by OS (Figure 4b-selected point 3 ). Chlorine and oxygen were minimally identified. Despite the low vacuum used for the EDS analyses do not provide images of high resolution, some micrometric white structures could be observed within the adhesive layer and at the initial portion of the resin tags. The detection of silicon in these regions (selected points 1 and 3 ) suggests that these structures are the microfillers of the adhesive. However, silicon was not detected in the hybrid layer (selected point 2) and in the medium third of the resin tag (selected point

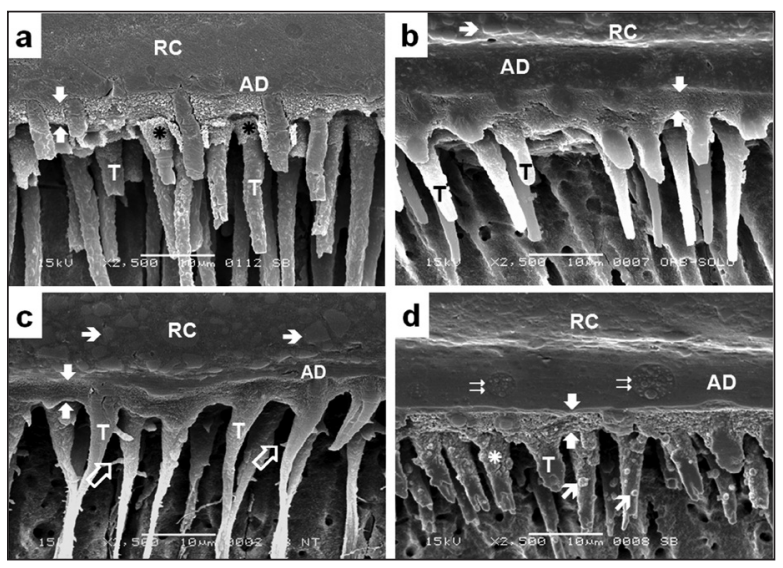

Figure 3. Representative SEM secondary electron image showing the micro-morphologic aspect of the bonding region produced by the adhesive systems Adper Single Bond (a), OptiBond Solo Plus (b), Prime \& Bond NT (c) and Adper Single Bond 2 (d) with dentin. All of them formed authentic hybrid layers with 4-5 $\mu$ m-thick (between white arrows) and resin tags (T) distributed along the interface. The hybridization process extended down to tubular walls, providing a morphologic aspect similar to the hybrid layer at the initial portion of some resin tags (asterisk). Lateral branches, which correspond to the secondary tubules, could be seen in some resin tags composed with Prime \& Bond NT (创). The hybrid layer formed by Adper Single Bond 2 showed an atypical granular aspect, and round grains $(\boldsymbol{x})$ could be observed

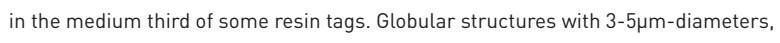
which were probably formed by the grouping of small "vesicles" of polyalkenoic acid $(\Longrightarrow)$, can be observed in the adhesive layer (AD) of SB2. Microfillers $(\rightarrow)$ of the resin composite $(\mathrm{RC})$ could be identified in some sections.

\begin{tabular}{lcccc}
\hline Adhesive Systems & Adper Single Bond & OptiBond Solo Plus & Prime \& Bond NT & Adper Single Bond 2 \\
\hline Bond Strength & $34.68 \pm 7.61 \mathrm{~b}$ & $33.33 \pm 5.64 \mathrm{~b}$ & $28.59 \pm 7.08 \mathrm{~b}$ & $42.64 \pm 11.44 \mathrm{a}$ \\
\hline
\end{tabular}

Means indicated by different letters are significantly different at $P<.05 ; n=25$ per group. 
4), suggesting that the microfillers didn't infiltrate within collagen network nor overstep the initial portion of the resin tags.

Calcium and phosphorus were predominant at the hybrid layer formed by NT (Figure 4c - selected point 2). Sodium and oxygen were minimally identified. No silicon signal was detected in the hybrid layer (selected point 2). Silicon signal was detected in the adhesive layer (selected point 1) and resin tag (selected point 3 and 4), suggesting the presence of nanofillers in these regions.
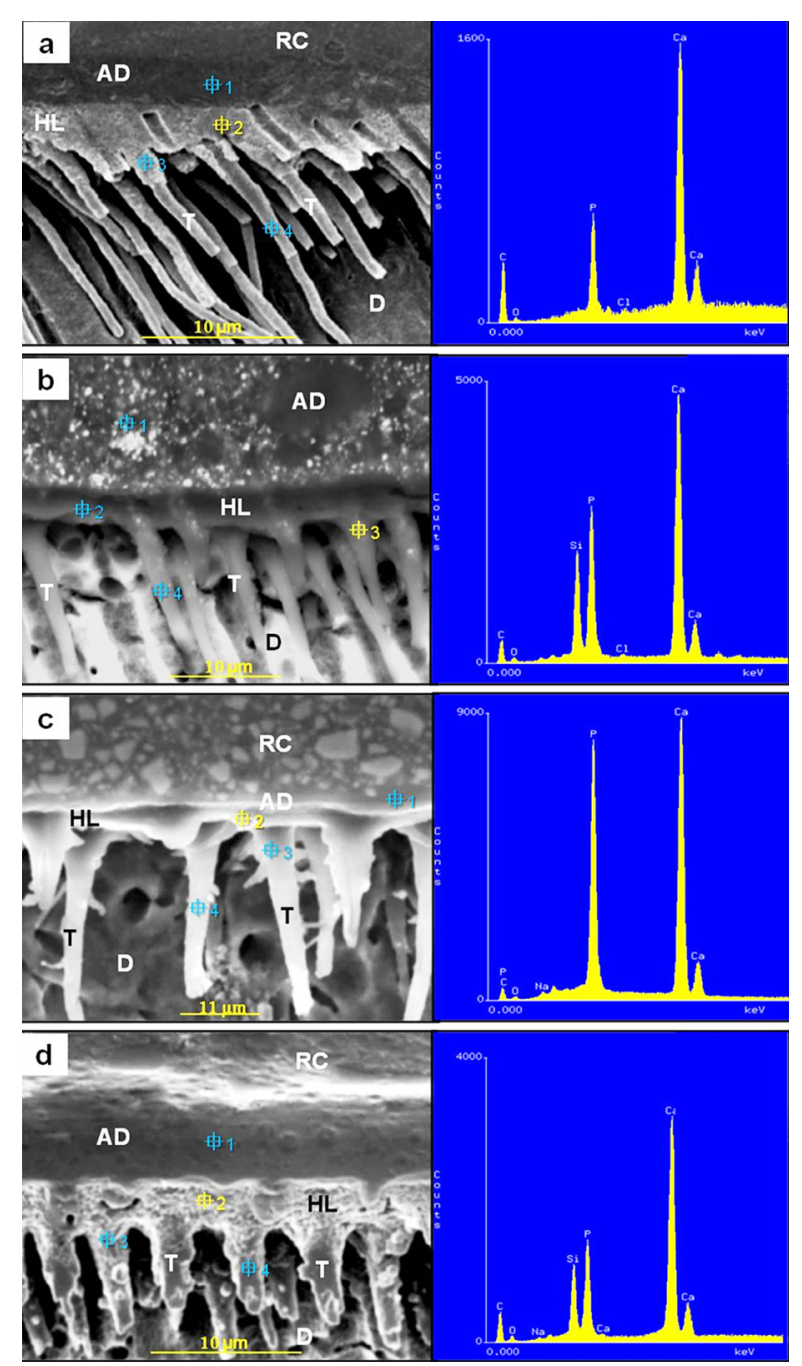

Figure 4. Representative SEM backscattered electron image of the EDS analysis of specific points selected at the resin-dentin interfaces formed by the adhesives evaluated. (a) Adper Single Bond (selected point 2) - the hybrid layer (HL) showed calcium as the main chemical element, followed by the phosphorus. Chlorine and oxygen were detected in reduced amounts; (b) OptiBond Solo Plus (selected point 3) - calcium and phosphorus were predominant at the initial portion of resin tag (T). Silicon is detected in expressive amount. Chlorine and oxygen were minimally identified; (c) Prime \& Bond NT (selected point 2) - calcium and phosphorus were predominantly detected at the HL. Sodium and oxygen were detected in reduced amounts. No signal for silicon was detected; (d) Adper Single Bond 2 (selected point 2) - the HL was predominantly constituted of calcium, followed by phosphorus. Silicon is detected in expressive amount. Weak signal of oxygen and sodium were detected. Carbon was detected in all the sections. (RC) - resin composite; (AD) Adhesive layer; (D) - Dentin.
The selected point 2, located at the hybrid layer formed by SB2, was predominantly constituted of calcium, followed by phosphorus (Figure $4 \mathrm{~d}$ ). Sodium and oxygen were minimally identified. Besides the selected point 2, silicon was detected in the other three selected points, suggesting the presence of nanofillers fillers inside the hybrid layer and resin tags.

Transmission electron microscopy analysis

Representative TEM micrographs of nondemineralized unstained sections from the bonding regions formed by the adhesive systems evaluated are illustrated (Figure 5). Figure 5a shows the bonding region of SB. A hybrid layer of approximately $5 \mu \mathrm{m}$-thick and resin tags could be identified. Some round formations, which probably represent the grouping of the polialkenoic acid
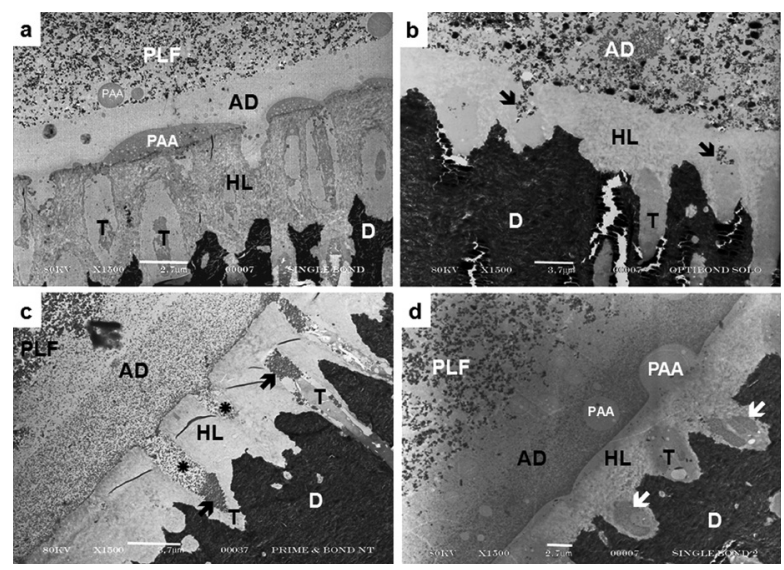

Figure 5. Representative TEM image showing nondemineralized unstained sections of the bonding regions formed by the adhesives studied with dentin (D). (a) Adper Single Bond, (b) OptiBond Solo Plus, (c) Prime \& Bond NT, (d) Adper Single Bond 2. Each of them formed uniform hybrid layers (HL) of approximately $5 \mu$ m-thick. The microfillers of the OptiBond Solo Plus are patently in the adhesive layer (AD) and in the initial portion of the dentinal tubules ( $\mathbf{*}$ ). Prime Bond NT exhibited nanofillers in $A D$ and infiltrated into the dentin tubules (asterisk), where they aggregate/ agglomerate, forming clusters ( $\boldsymbol{\|}$ ). The nanofillers of Adper Single Bond 2 were uniformly distributed in $\mathrm{AD}$ and inside dentinal tubules ( $\mathbf{(}$ ), composing resin tags (T) with inorganic material. They can also be identified in the $\mathrm{HL}$ in a non-agglomerated status, however less concentrated than in the other regions. (PLF) - Flowable resin composite Clearfil Protect Liner F; (PAA) - Polyalkenoic acid.
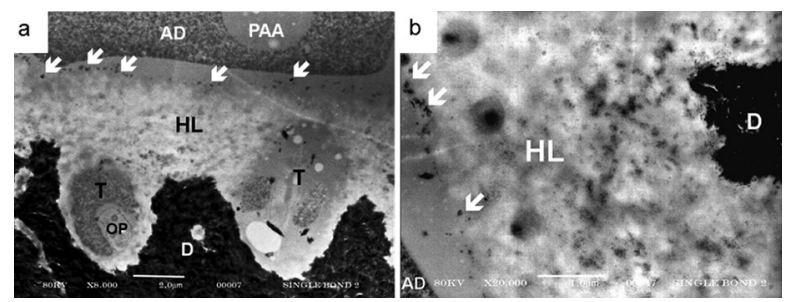

Figure 6. Representative TEM image showing nondemineralized, unstained sections of the hybrid layer produced by Adper Single Bond 2. At 8.000X (a) and 20.000X (b) magnifications, it becomes possible to better distinguish the nanofillers dispersed in the hybrid layer (HL). Some micrometric structures ( $\mathbf{(})$, which are probably fillers used as thickener for the phosphoric acid etcher, were retained on the top of the hybrid layer. (AD) - Adhesive; (D) - Dentin. (T) Resin tags; (PAA) - Polyalkenoic acid. (OP) - Odontoblast process. 
molecules, were observed in the adhesive layer.

The bonding region formed by $\mathrm{OS}$ is illustrated in Figure 5b. The hybrid layer exhibits, approximately $5 \mu \mathrm{m}$ of thickness. Microfillers could be discerned in the adhesive layer and at the initial portion of the dentinal tubules, forming particlereinforced resin tags. However, these particles were not present in the interfibrillar spaces of the hybrid layer, which were only infiltrated by the resin monomers of the adhesive.

Figure $5 c$ shows the bonding region formed by NT. The adhesive layer is characterized by the presence of nanofillers. They penetrated some of the exposed tubules, composing reinforced resin tags. The hybrid layer presents approximately $5 \mu \mathrm{m}$ of thickness, where nanofillers were not observed. The dentinal tubules are also affected by the hybridization process, forming funnel-shaped, hybridized tubular walls. There was a tendency of the nanofillers to aggregate/agglomerate, forming electron-dense micrometric structures called clusters. These structures could be observed within the adhesive layer and were concentrated in dentinal tubules.

The interaction of SB2 with dentin is illustrated in Figure $5 \mathrm{~d}$. The hybrid layer was approximately $5 \mu \mathrm{m}$ thick. Some globular structures, similar to those found in SB analysis and thought to be grouping of the methacrylated-polyalkenoic acid molecules, were identified in the adhesive layer. Nanofillers were uniformly distributed throughout the entire extension of the adhesive layer, hybrid layer and in the dentinal tubules. The concentration and distribution of nanofillers inside dentinal tubules and in the adhesive layer are similar and higher than those observed in the hybrid layer, where the nanofillers were intermixed with a collagen matrix network. At higher magnifications (Figure 6a and 6b), it was possible to better distinguish the nanofillers dispersed within the collagen fibrils. Some micrometric structures, probably silica particles used as thickener for the phosphoric acid etchant, were retained at the top of the hybrid layer.

According to these results, the hypothesis tested is rejected. The adhesives evaluated showed particular interaction morphology with dentin, and the bond strength of SB2 was significantly higher than that of obtained by the other materials.

\section{DISCUSSION}

The bonding systems evaluated were considered effective, forming authentic hybrid layers (Figure 3 and 5). Similar results were described in previous studies, ${ }^{18-21}$ what contributes to fundament the clinical indication of the simplified-step adhesives evaluated.

The analysis of the $\mu$-TBS results showed that OS, NT and SB had no statistical differences. These data attest that the presence of microffilers in OS and nanofillers in NT did not determine superior $\mu$-TBS to dentin. Using similar methodology, other authors ${ }^{12,22-23}$ also obtained similar bond strength values among adhesives with/or without filler. However, the present study showed that SB2, which only modification in relation to its predecessor (SB) was the incorporation of nanofillers (Table 1), reached significantly higher bond strength compared with the others adhesives evaluated (Table 2). On the other hand, similar influence of the nanofillers in terms of the bond strength was not verified by Nunes et al $^{13}$ These authors obtained statically similar mean bond strength values between NT and an experimental version without nanofillers. They still described that the incorporation of microfillers to compose a modification of $\mathrm{SB}$ resulted in significant reduction of the bond strength in comparison with the original adhesive. Due to these antagonistic results, it was evident that the incorporation of fillers in simplified-step adhesives can influence the bond strength to dentin only under special conditions. Furthermore, the mechanism that establishes this control is determined, probably, by the characteristics of the fillers and their interaction with dentin.

To better understand the action of the inorganic fillers in this process, the chemical elements that compose the resin-dentin interfaces were assessed using SEM/EDS. In a general way, the fillers of the adhesives were not clearly distinguished with SEM/EDS analysis that operates in low vacuum mode and carbon coated specimens, resulting in images with modest definition. However, it is relevant to emphasize that the EDS analysis using SEM allows a more reliable microanalysis of the resin-dentin interface in comparison to the EDS analysis performed by TEM. In this case, the detection of Si could be questioned, since the elemental mapping of the polymerized sections of epoxy resin used for laboratory embedding pro- 
cedures for TEM examination could reveal the presence of Si. ${ }^{11}$ As the epoxy resin infiltrates into spaces that were depleted following the laboratory dehydration of the specimens, the detection of $\mathrm{Si}$ could be due to the presence of the epoxy resin and not from the structure analyzed, which would disqualify the results.

The detection of expressive amounts of calcium and phosphorus in the hybrid layers formed by the adhesives SB, NT and SB2 (Figure 4a, 4c and $4 \mathrm{~d}$ ), and at the initial portion of the resin tag of the adhesive OS (Figure 4b), was expected and observed. These chemical elements form the apatite crystals, which are the primary inorganic components of the dentin. ${ }^{24}$ They were identified due to their permanence in some regions of the dentin that were not completely devoid of the minerals, even after the phosphoric acid etching. Furthermore, an insoluble calcium phosphate complex may remain in the tissue even after the water rinsing of the etching agent. Especially for OS and NT, the detection of phosphorus in its bonding regions can also be attributed to the organic composition of these materials. The phosphate monomers glycerol phosphate dimethacrylate (GPDM) and dipentaerythritolpentaacrylate monophosphate (PENTA) are present in the composition of OS and NT, respectively. The chemical elements sodium, oxygen, and chlorine, which were revealed in some of the analyses, may represent residues of the sodium hypochlorite ( $\mathrm{NaOCl}$ ) solution, used as a deproteinization agent to remove the organic phase of the specimens during its processing for EDS analysis. Carbon, employed to cover the specimens and provide electronic conductance for the elemental analysis using SEM, was present in each of the analyses (Figure 4). The presence of other chemical elements at the resin-dentin interfaces should not be disregarded. Probably, they were present in such low concentrations that they could not be identified in this process.

To complement the study of the resin-dentin bonding interfaces, it was realized that a micromorphologic characterization of nondemineralized unstained sections using TEM was necessary. Although the micromophologic aspects of the bonding regions could be better revealed with the use of stained sections, the authors preferred to process unstained sections so as to avoid the formation of nonspecific electrondense staining, located at the periphery of the collagen fibrils, as observed in a previous study using TEM sections. ${ }^{11}$ This electrondense staining could represent remnants of the proteoglycans and noncollagenous proteins that were retained within the collagen network after the acid conditioning of dentin, ${ }^{25-26}$ and could be mistaken for the nanofillers of the adhesives. Without the interference of these stained "collagen adjuncts", 27 the study of the interaction between the adhesives and dentin was viable with the patent observation of the inorganic fillers. When associated with the SEM/EDS analysis, it can be affirmed that the structures distributed in the adhesive layer and inside the dentinal tubules at the bonding interface of OS and NT (Figure $5 b$ and $5 c$ ) are inorganic fillers from the adhesives. Likewise, the presence of nanofillers in the resin tags, adhesive layer and in the hybrid layer produced by SB2 (Figure 5d, 6a, and 6b), could be confirmed.

The infiltration of nanofillers within the collagen fibrils is thought to be the main and decisive factor for the $\mu$-TBS values reached by SB2. According to this concept, after light-curing, the treated nanofillers would bond with the resin matrix of the adhesive and thereby act as a strengthening element for the severely demineralized dentin, replacing the solubilized apatite filler crystallites. As a result, this structural condition would provide an improvement of the mechanical properties of the SB2-hybrid layer when compared with the hybrid layers produced by the other adhesives. This can partially explain the smallest incidence of cohesive fractures in the hybrid layer (Type III) observed with SB2, as demonstrated by SEM analysis of the failure modes after $\mu$-TBS test (Figure 2). Moreover, the higher percentage of cohesive failure in the adhesive (Type I) for SB2 (Figure 2), suggests that the mechanical properties of the material was not improved and is in agreement with the results of previous study. ${ }^{28}$ So, the condition of strengthening of the hybrid layer by nanofillers was reaffirmed to explain the increased bonding effectiveness.

As suggested by the interpretation of the results, the infiltration of inorganic fillers within the collagen fibrils is essential for the bond strength improvement. Therefore, to obtain this interaction form, three issues must be considered, as described by Tay et al ${ }^{11}$ First, the fillers should be sufficiently small (nanofillers) to infiltrate within the interfibrillar spaces of the demineralized dentin, which are 
about $12 \mathrm{~nm}$ in width. ${ }^{10} \mathrm{As}$ well, the nanofillers must be physically and chemically stabilized to avoid aggregation/agglomeration during the storage and/ or application of the bond solution, which creates micrometric structures called "clusters" that are too large to infiltrate collagen fibrils. " Second, as the molecular weight of the nanofillers and the resin monomers of the adhesives differ markedly, the resulting diffusion rate is dissimilar. This could preclude the complete infiltration of the nanofillers within the collagen fibrils, even if the interfibrillar spaces were maintained fully extended by the correct application of the wet-bonding technique. ${ }^{29}$ Third, the infiltration of the nanofillers could be physically impeded by the presence of a hydrogel of residual ground substances, proteoglycans and noncollagenous proteins in the interfibrillar spaces of the demineralized collagen network. ${ }^{27,30}$

Considering such premises, some considerations regarding the bonding systems analyzed in this study should be addressed. In relation to the inorganic portion of the adhesives, OS have fillers composed predominantly of barium aluminoborosilicate and sodium hexafluorosilicate in the order of $0.4 \mu \mathrm{m}$. This dimension disables them from penetrating the nanometric spaces within the collagen fibrils to compose the hybrid layer. Consequently, they remain in the adhesive layer and at the initial portion of the dentinal tubules (Figure $4 \mathrm{~b}$ and $5 \mathrm{~b}$ ). This way, the particles form an inorganicreinforced polymer network that may serve as a shock-absorbing layer of intermediate modulus of elasticity between the hybrid layer and the resin composite, in accordance with the elastic cavity wall concept of Kemp-Scholte and Davidson. ${ }^{31}$ However, they assume irrelevant importance in terms of bond strength, which is in agreement with the results of other studies. ${ }^{12,22-23}$ The simplifiedstep bonding system, in spite of representing an operational simplification, needs complex chemical formulation to combine hydrophilic and hydrophobic monomers into a single bottle. Therefore, characteristics regarding the chemical balance of the adhesive, the organic solvent employed, ${ }^{3}$ and operatory aspects for the bonding procedures as related to the degree of dentin moisture after acid etching, acquire larger relevance in terms of determining the bonding effectiveness. ${ }^{32}$

The nanofillers of the adhesives NT and SB2, are constituted of synthetic silica and produced by flame pyrogenic or sol-gel techniques, respectively. ${ }^{33}$ The mean primary particle size ranged from 7 to $40 \mathrm{~nm}$ for NT11 and $5 \mathrm{~nm}$ (manufacturer's information) for SB2. In the case of the fumed silica used for NT, there is a natural tendency of the primary nanoparticles to aggregate/agglomerate forming branched and chainlike three-dimensional clusters as a result of internal interparticle van der Waal's forces. This electrostatic attraction forces are nearly $10^{36}$ more intense than the gravitational force in the nanoscale. ${ }^{34-35}$ According to the Derjaguim-Landau-Vermey-Overbeek (DLVO) theory, the control of the aggregation/agglomeration phenomena when preparing nanoparticles may be accomplished by the inclusion of surface modifiers, which provides electrostatic repulsion between the charged surfaces. ${ }^{36-37}$ However, the tendency of the nanofillers to aggregate/agglomerate can be further intensified, as a result of the surface treatment. This can occurs due to the formation of silanol groups on the surface of the modified particles, resulting in hydrogen bridge linkages with adjacent particles ${ }^{38}$ that impede their infiltration within the collagen fibrils. Another adverse condition for the infiltration of the nanofillers to compound the hybrid layer is the possibility of a hydrogen bridge formation between silica and amino acids moieties of the collagen. This possibility was based on the observation that similar interactions may occur among silica fillers, used as thickeners in acid conditioners, and proteins of the collagen network. ${ }^{11,39}$ In this case, the silica was retained on the top of the hybrid layer, even after thorough rinsing with water, as suggested by the Figure 6 .

To overcome these impediments, the manufacturers of dental materials, using the recent progresses regarding nanoscience and nanomanipulation, modify the surface of the nanofillers with a group of molecules known as silane agents. These molecules are bifunctional, containing alkoxy-silane groups at one end, which react and covalently bond with the silanol groups, and methacrylatefunctinality at the other, that are available for copolymerization with the resin monomers of the adhesive. ${ }^{40-41}$ Thus, approximately $70 \%$ of the silanol groups on the surface of the synthesized nanoparticles were substituted with siloxane groups..$^{42} \mathrm{As}$ a consequence, the nanofillers acquire a hydrophobic character, becoming more easily dispersed in colloidal solution, ${ }^{40,42}$ and remaining un-agglom- 
erated as observed for SB2. Besides the physicalchemical stabilization, this surface modification allows the linkage between nanofillers and the resin monomers of the adhesives, forming "nanomers", which are constituted of nanofillers and organic components. ${ }^{38}$

Even after the treatment with silane, the nanofillers of the NT do not maintain the physical-chemical stability during the application of the material, forming clusters (Figure $5 \mathrm{c}$ ). As described by Tay et $\mathrm{al}^{11}{ }^{11}$ the dispersion into individual primary particles was not observed, due in part to the incomplete chemical stabilization of the particles treated with silane, allowing the bonding with the residual hydrogen. Additionally, the destabilization of the electrostatic forces of the nanofillers may be intensified as a consequence of alterations in the $\mathrm{pH}$, ionic strength and/or loss of the solvent. All of these variations can occur during the adhesive application, contributing to the nanofillers overcoming the critical radius for repulsion from the Stern's potential. ${ }^{36,38}$ Especially for NT, the loss of the acetone, a highly volatile compound used as a solvent, might also contribute to destabilization of the nanofillers. The incorporation of resin monomers in acetone is chemically complex, resulting in adhesives constituted of approximately $80 \%$ (volume) of solvent and only $20 \%$ (volume) of monomers. ${ }^{43}$ With the acetone volatilization, this proportional relationship is severely modified, which can change the $\mathrm{pH}$ and the chemical interaction among the molecules, contributing for the destabilization of the electrostatic forces in the surface of the nanofillers. As a consequence, there is a reduction of the potential energy barrier of the silane and the nanofillers, which is closely approximate forming clusters. This way, the particles of NT carry out similar functions to the micrometric fillers present in other adhesives as, for instance OS, forming then an elastic cavity wall, ${ }^{31}$ but failing to strengthen the hybrid layer.

In contrast with the previous case, the nanofillers of SB2 infiltrated within collagen fibrils to form the hybrid layer (Figure 4d, 5d, and 6). Special characteristics acquired for these nanofillers during manufacture allowed them to stay uniformly dispersed, even during the application of the adhesive. The physical-chemical stability of these structures was probably obtained with an improvement of the surface treatment with silanes, after the primary particles of synthetic silica $(5 \mathrm{~nm})$ were produced by sol-gel technique. ${ }^{33,42}$ Chaimberg et $\mathrm{al}^{44}$ have reported that the amount of silane coupling agent chemisorbed on oxide supports differs drastically if changing the surface modification procedures. The type of solvents, $\mathrm{pH}$, and amount of absorbed water on particles largely affected the chemisorbed content of silane. ${ }^{33,44}$ One example of improvement in the treatment of inorganic particles was recently described by lijima and Kamiya, ${ }^{45}$ who modified the surface of silica nanoparticles with slight amounts of additional $\mathrm{pH}$ controlled water. It was found that when 3-glycidoxypropyltrimethoxysilane was modified with the slight addition of acidic water, a relatively large steric repulsive force was measured with a colloid probe atomic force microscopy method, while the mixture modified with the slight addition of base water possessed a small steric repulsive force. Therefore, the authors of the present study consider that a similar approach could have been performed to provide larger reactivity between silane coupling agents and the surface of the nanofillers, resulting in a more efficient "chemical isolation". Moreover, the water/ ethanol solvent employed for SB2 is less critical regarding the chemical stability of the adhesive. ${ }^{46}$ The possible difficulty of the uniform infiltration of the nanofillers within the collagen fibrils due to its smaller diffusion rate compared with that of the resin monomers, ${ }^{29}$ was not observed for SB2. The chemical stability reached by this adhesive, as described above, preserved the covalent bond formed between the nanofillers and the resin monomers by the silane. With the preservation of this primary chemical bond, the nanofillers were carried within the collagen fibrils bonded with the organic portion of the adhesive. Thus, the difference in the diffusion rates assumes irrelevant importance for this aspect.

The presence of the nanofillers may transpose biophysical barriers, which are formed in the course within the collagen fibrils, to reach the whole extension of the hybrid layer. The organic matrix of the demineralized dentin as substrate for the adhesion is considered, in general, a network matrix mainly composed of collagen fibrils sustained by the water. Inside this structure, residual substances such as proteoglycans and noncollagenous proteins were also identified. ${ }^{27,} 30$ It was observed that after dentin demineralization, just a part of the complex of noncollagenous proteins is solubilized and extracted by the tissue for the water rinsing. The other portion is grouped together to form a dense population of granules, with approximately 1.5 to $2 \mathrm{~nm}$ of diameter, along the collagen fibrils. ${ }^{47}$ 
In spite of these physical barriers, there is enough space for the movement of the dispersed nanofillers within the interfibrillar spaces, which are about $12 \mathrm{~nm}$ in width. ${ }^{10}$ As the physical-chemical stability of the nanofillers was not disordered, chemical interactions with the remaining proteoglycans, although highly loaded for polianions, was inhibited. For these reasons, the nanofillers of SB2 were able to infiltrate the whole extension of the hybrid layer.

The presence of nanofillers in the hybrid layer enables the concept of strengthening of the dentin that has been extensively demineralized by the acid etching. This innovation is a result of the improvement in nanomaterials preparation that has been performed in the last years and point to a new direction for the adhesive systems. The development of the nanoscale science is still in an early stage and it is expected that further applications will appear for dental materials in the near future.

\section{CONCLUSIONS}

Within the limitations of this study, it can be concluded that:

The infiltration of nanofillers within the interfibrillar spaces of the etched dentin strengthens the hybrid layer, improving the bonding effectiveness.

\section{ACKNOWLEDGMENTS}

The authors are grateful to Mr. Adriano Luis Martins and Mrs. Eliene Aparecida Orsini Narvaes for technical support. This study was supported, in part, by grants from CAPES (P.I. Vinicius Di Hipólito).

\section{REFERENCES}

1. Van Meerbeek B, Dhem A, Goret-Nicaise M, Braem M, Lambrechts $P$, VanHerle G. Comparative SEM and TEM examination of the ultrastructure of the resin-dentin interdiffusion zone. J Dent Res 1993;72:495-501.

2. Van Meerbeek $B$, Inokoshi $S, B r a e m ~ M$, Lambrechts $P$, Vanherle $G$. Morphological aspects of the resin-dentin interdiffusion zone with different dentin adhesive systems. $J$ Dent Res 1992;71:1530-1540.

3. Pashley DH, Tay FR, Breschi L, Tjäderhane L, Carvalho RM, Carrilho M, Tezvergil-Mutluay A. State of the art etch-andrinse adhesives. Dent Mater 2011;27:1-16.

4. Van Meerbeek B, Yoshihara K, Yoshida Y, Mine A, J de M, K L VL.. State of the art of self-etch adhesives. Dent Mater 2011;27:17-28.
5. Sano H, Takatsu T, Ciucchi B, Russell CM, Pashley DH. Tensile properties of resin-infiltrated demineralized human dentin. J Dent Res 1995;74:1093-1102.

6. Maciel KT, Carvalho RM, Ringle RD, Preston CD, Russell CM, Pashley DH. The effects of acetone, ethanol, HEMA, and air on the stiffness of human decalcified dentin matrix. $J$ Dent Res 1996;75:1851-1858.

7. Zhang Y, Agee K, Nör J, Carvalho R, Sachar B, Russell C, Pashley D. Effects of acid-etching on the tensile properties of demineralized dentin matrix. Dent Mater 1998;14:222-228.

8. Pashley DH, Ciucchi B, Sano H, Carvalho RM, Russell CM. Bond strength versus dentine structure: a modelling approach. Arch Oral Biol 1995;40:1109-1118.

9. Van Meerbeek B, Conn LJ, Jr., Duke ES, Eick JD, Robinson SJ, Guerrero D. Correlative transmission electron microscopy examination of nondemineralized and demineralized resin-dentin interfaces formed by two dentin adhesive systems. J Dent Res 1996;75:879-888.

10. Osorio E, Toledano M, Aguilera FS, Tay FR, Osorio R. Ethanol wet-bonding technique sensitivity assessed by AFM. J Dent Res 2010;89:1264-1269.

11. Tay FR, Moulding KM, Pashley DH. Distribution of nanofillers from a simplified-step adhesive in acid-conditioned dentin. $J$ Adhes Dent 1999;1:103-117.

12. Braga RR, Cesar PF, Gonzaga CC. Tensile bond strength of filled and unfilled adhesives to dentin. Am J Dent 2000;13:7376.

13. Nunes MF, Swift EJ, Perdigao J. Effects of adhesive composition on microtensile bond strength to human dentin. Am J Dent 2001;14:340-343.

14. Perdigao J, Geraldeli S. Bonding characteristics of selfetching adhesives to intact versus prepared enamel. $J$ Esthet Restor Dent 2003;15:32-41; discussion 42.

15. Shinohara MS, De Goes MF, Schneider LF, Ferracane JL, Pereira PN, Di Hipólito V, Nikaido T. Fluoride-containing adhesive: durability on dentin bonding. Dent Mater 2009;25:1383-1391.

16. Inokoshi S, Hosoda H, Harnirattisai C, Shimada Y, Hosoda H. A study on resin-impregnated layer of dentin. Part I. A comparative study on the decalcified and undecalcified sections and the application of argon beam ion etching to disclose the resin impregnated layer of dentin. Jpn J Conserv Dent 1990;33:427-442.

17. Perdigao J, Lambrechts P, Van Meerbeek B, Vanherle G, Lopes AL. Field emission SEM comparison of four postfixation drying techniques for human dentin. $J$ Biomed Mater Res1995;29:1111-1120.

18. Perdigao J, Lopes MM, Gomes G. In vitro bonding performance of self-etch adhesives: II-ultramorphological evaluation. Oper Dent 2008;33:534-549. 
19. Elkassas D, Taher HA, Elsahn N, Hafez R, El-Badrawy W. Effect of the number of applications of acetone-based adhesives on microtensile bond strength and the hybrid layer. Oper Dent 2009;34:688-696.

20. Teixeira CS, Chain MC. Evaluation of shear bond strength between self-etching adhesive systems and dentin and analysis of the resin-dentin interface. Gen Dent 2010;58:e5261.

21. Waidyasekera PG, Nikaido T, Weerasinghe DD, Tagami J. Bonding of acid-etch and self-etch adhesives to human fluorosed dentine. J Dent 2007;35:915-922.

22. Can Say E, Nakajima M, Senawongse P, Soyman M, Ozer F, Ogata M, Tagami J. Microtensile bond strength of a filled vs unfilled adhesive to dentin using self-etch and total-etch technique. J Dent 2006;34:283-291.

23. Lee YK, Pinzon LM, O'Keefe KL, Powers JM. Effect of filler addition on the bonding parameters of dentin bonding adhesives bonded to human dentin. Am J Dent 2006;19:23-27.

24. Marshall GW, Jr., Marshall SJ, Kinney JH, Balooch M. The dentin substrate: structure and properties related to bonding. J Dent 1997;25:441-458.

25. Glimcher MJ, Lefteriou B, Kossiva D. On the problem of covalent linkages between phosphoproteins and collagen in bovine dentin and bone. J Bone Miner Res 1986;1:509-522.

26. Goldberg M, Takagi M. Dentine proteoglycans: composition, ultrastructure and functions. Histochem $J$ 1993;25:781-806.

27. Linde A, Jontell M, Lundgren T, Nilson B, Svanberg U. Noncollagenous proteins of rat compact bone. $J$ Biol Chem 1983;258:1698-1705

28. Giannini M, Mettenburg D, Arrais CA, Rueggeberg FA. The effect of filler addition on biaxial flexure strength and modulus of commercial dentin bonding systems. Quintessence Int 2011;42:e39-43.

29. Eick JD, Robinson SJ, Byerley TJ, Chappell RP, Spencer P, Chappelow CC. Scanning transmission electron microscopy/energy-dispersive spectroscopy analysis of the dentin adhesive interface using a labeled 2-hydroxyethylmethacrylate analogue. J Dent Res 1995;74:1246-1252.

30. Linden LA, Kallskog O, Wolgast M. Human dentine as a hydrogel. Arch Oral Biol 1995;40:991-1004.

31. Kemp-Scholte CM, Davidson CL. Marginal integrity related to bond strength and strain capacity of composite resin restorative systems. J Prosthet Dent 1990;64:658-664.

32. Perdigao J. Dentin bonding-variables related to the clinical situation and the substrate treatment. Dent Mater 2010;26:e24-37.

33. Moszner N, Gianasmidis A, Klapdohr S, Fischer UK, Rheinberger V. Sol-gel materials 2. Light-curing dental composites based on ormocers of cross-linking alkoxysilane methacrylates and further nano-components. Dent Mater $2008 ; 24: 851-856$.
34. Roco MC. Reviews of national research programs in nanoparticle and nanotechnology research in the U.S.A. $J$ Aerosol Sci 1995;32:749-760.

35. Seeman NC. DNA nanotechnology. Materials Today 2003;6:24-39.

36. Dickinson E. An introduction to food colloids. New York: Oxford University Press. 1992:174-199.

37. Horisberger M. Colloidal gold: a cytochemical marker for light and fluorescent microscopy and for transmission and scanning electron microscopy. In: Albrecht RM, Hodges GM (eds). Biotechnology and bioapplications of colloidal gold. Chicago, IL: Scanning Microscopy International 1988;19-40.

38. Schmidt HK, Oliveira PW, Krug H. Hybrid sols as intermediates to inorganic-organic. nanocomposites. In: Coltrain BK, Sanchez C, Schaefer DW, Wilkes G (eds). Better ceramics through chemistry VII: organic/inorganic hybrid materials. Mat Res Soc Symp Proc 1996;435(13-24.).

39. Perdigao J, Lambrechts $P$, van Meerbeek B, Tome AR, Vanherle G, Lopes AB. Morphological field emission-SEM study of the effect of six phosphoric acid etching agents on human dentin. Dent Mater 1996;12:262-271.

40. D'Agostino A, Errico ME, Malinconico M, De Rosa M, Avella $M$, Schiraldi C. Development of nanocomposite based on hydroxyethylmethacrylate and functionalized fumed silica: mechanical, chemico-physical and biological characterization. J Mater Sci Mater Med 2011;22:481-490.

41. Mitra SB, Wu D, Holmes BN. An application of nanotechnology in advanced dental materials. $J$ Am Dent Assoc 2003;134:1382-1390.

42. Michael G, Fench $H$. Basic characteristics of AEROSIL $®$ Bulletin Pigments. Number 11. 4th Edition. Frankfurt, Germany: Degussa AG. 1993:5-80.

43. Reis AF, Oliveira MT, Giannini M, De Goes MF, Rueggeberg FA. The effect of organic solvents on one-bottle adhesives' bond strength to enamel and dentin. Oper Dent 2003;28:700706.

44. Chaimberg M, Cohen Y. Note on the silylation of inorganic oxide supports. J Colloid Interface Sci 1990;134:576-579.

45. lijima M, Tsukada M, Kamiya H. Effect of surface interaction of silica nanoparticles modified by silane coupling agents on viscosity of methylethylketone suspension. J Colloid Interface Sci 2007;305:315-323.

46. Malacarne-Zanon J, de Andrade E Silva SM, Wang L, de Goes MF, Martins AL, Narvaes-Romani EO, Anido-Anido A, Carrilho MR. Permeability of dental adhesives - A SEM assessment. Eur J Dent 2010;4:429-439.

47. Goldberg M, Septier D. Visualization of proteoglycans and membrane-associated components in rat incisor predentine and dentine using ruthenium hexammine trichloride. Arch Oral Biol 1986;31:205-212. 